BRX-TH-335

\title{
Gravity Theories with Lightlike Sources in $D=3$
}

\author{
S. Deser \\ Alan R. Steif \\ Brandeis University \\ Department of Physics \\ Waltham, MA 02254 \\ deser@binah.cc.brandeis.edu \\ steif@binah.cc.brandeis.edu
}

\begin{abstract}
Spacetimes generated by a lightlike particle source for topologically massive gravity and its limits - Einstein gravity and the pure gravitational Chern-Simons model are obtained both by solving the field equations and by infinite boosts of static metrics. The resulting geometries are the first known solutions of topologically massive gravity that are asymptotically flat and generated by compact matter sources. Explicit metrics describing various multiphoton solutions are also derived. For Einstein gravity, we also construct such solutions by null boost identifications of Minkowski space and thereby obtain limits on the energies of the sources.
\end{abstract}




\section{Introduction.}

There has been renewed interest in geometries generated by a lightlike particle in general relativity because of their relevance to quantum scattering of two gravitationally coupled particles at Planckian energies [1]. Three-dimensional gravity theories provide a simplified, but interesting, arena to study these questions: in Einstein gravity and (conformally invariant) Chern-Simons gravity (CSG) there are no field degrees of freedom at all, while the more general topologically massive gravity (TMG) [2] is dynamical, yet very different from $\mathrm{D}=4$ gravity. We propose here to solve for and interpret the geometries generated by lightlike matter sources (which we call "photons" for short), as a first step towards studying the corresponding quantum scattering problems in these systems [3]. In general, photons are described by plane-fronted wave spacetimes. We will exploit the fact that such metrics "linearize" the field equations (so that the linear and full curvature tensors coincide) to obtain a wide class of solutions. In particular, we will find, for full nonlinear TMG, the metric generated by a photon; this is the first known example of an asymptotically flat solution with a localized source in the theory. Indeed, in TMG only the linearized "Schwarzschild" solution is known for a static particle; in the limit of infinite boost it will be shown to coincide with the exact plane wave metric. We will also display multiphoton solutions in these models, and relate the geometrical aspects of our results to those discussed in [4] for pure Einstein gravity.

\section{The Single Photon Solution.}

The plane-fronted wave ansatz in $D$ spacetime dimensions,

$$
d s^{2}=d s_{0}^{2}+F(u, \vec{y}) d u^{2}, \quad d s_{0}^{2} \equiv-d u d v+d \vec{y}^{2},
$$

simplifies the Einstein tensor to the linearized form

$$
G_{\mu \nu}=-\frac{1}{2} \nabla_{T}^{2} F \quad l_{\mu} l_{\nu}, \quad l_{\mu} \equiv \partial_{\mu} u
$$

Our sign convention is $R_{\mu \nu} \sim+\partial_{\alpha} \Gamma_{\mu \nu}^{\alpha}, \vec{y}$ are the $D-2$ transverse coordinates, $u=t-x$ and $v=t+x$ are the usual lightcone coordinates, and $\nabla_{T}^{2}$ is the flat space Laplacian in the transverse dimensions. Henceforth, we specialize to $D=3$ and denote $y$-differentiation by a

prime. Thus, only the component $G_{u u}=-\frac{1}{2} F^{\prime \prime}$, or equivalently $R_{u y u y}$ (since the Riemann 
and Einstein tensors are double duals of one another) fails to vanish. We shall also need the Cotton (Weyl) tensor, $C^{\mu \nu} \equiv \epsilon^{\mu \alpha \beta} D_{\alpha}\left(R_{\beta}{ }^{\nu}-\frac{1}{4} \delta_{\beta}^{\nu} R\right)$; it too simplifies drastically:

$$
C_{\mu \nu}=-\frac{1}{2} F^{\prime \prime \prime} l_{\mu} l_{\nu}
$$

where our volume form convention is $\epsilon^{t x y}=1$. The (parity violating) field equations of TMG [2] are

$$
E_{\mu \nu} \equiv G_{\mu \nu}+\frac{1}{\mu} C_{\mu \nu}=-\kappa^{2} T_{\mu \nu}
$$

where $\kappa^{2}$ is the Einstein constant with dimensions of inverse mass and $\mu$ is the graviton's mass or, in our classical framework, its inverse range. For simplicity of notation we take $\mu>0$; the theory with opposite sign choice is parity conjugate to (2.4) and goes through analogously. Note that TMG reduces to Einstein gravity* as $\mu \rightarrow \infty$, and to CSG as $\mu \rightarrow 0, \kappa^{2} \rightarrow \infty$ with $\kappa^{2} \mu$ fixed.

For our plane wave ansatz and with a right-moving photon source of energy $E$, (2.4) reduces to just its $u$ component,

$$
\left(1+\frac{1}{\mu} \frac{\partial}{\partial y}\right) F^{\prime \prime}=2 \kappa^{2} E \delta(y) \delta(u)
$$

Hence the curvature is

$$
G_{u u}=R_{u y u y}=-\frac{1}{2} F^{\prime \prime}=-\mu \kappa^{2} E e^{-\mu y} \theta(y) \delta(u)+A(u) e^{-\mu y} .
$$

We will drop the homogeneous solution, $A e^{-\mu y}$, to maintain asymptotic flatness. From (2.6) we see that the spacetime is flat off the $u=0$ plane and for negative $y$. Integrating (2.6) yields the metric**

$$
F=2 \kappa^{2} E f(y) \delta(u)+B y+C, \quad f=\left(y+\frac{1}{\mu}\left(e^{-\mu y}-1\right)\right) \theta(y) .
$$

Here $f$ can be viewed as an inverse of the operator $\left(1+\frac{1}{\mu} \frac{\partial}{\partial y}\right)\left(\frac{\partial}{\partial y}\right)^{2}$; the homogeneous solutions involve arbitrary functions $B(u), C(u)$ that can be removed by the coordinate transformation

$$
\begin{aligned}
& u \rightarrow u \\
& v \rightarrow v+b y-\frac{1}{4} \int_{0}^{u} b^{2}+\frac{1}{2} b \int_{0}^{u} b+c \\
& y \rightarrow y+\frac{1}{2} \int_{0}^{u} b
\end{aligned}
$$

\footnotetext{
* More precisely, it reduces to "ghost" Einstein gravity, with the opposite sign of $\kappa^{2}$ than that required in $D=4$; this sign choice is forced by requiring TMG to be non-ghost [2].

** This method of solving (2.4) was found independently by J. McCarthy; see [3].
} 
where $b(u)=\int_{0}^{u} B$ and $c(u)=\int_{0}^{u} C$. If we make a further coordinate transformation to remove the $\delta(u)$ factor in $F, \tilde{v}=v-2 \kappa^{2} E f(y) \theta(u)$, then the metric (2.1) with (2.7) takes the form

$$
\begin{aligned}
d s^{2} & =-d u d \tilde{v}+d y^{2}-2 \kappa^{2} E \theta(u) f^{\prime}(y) d u d y \\
& =\theta(u)\left\{-d u d\left(\tilde{v}+2 \kappa^{2} E f(y)\right)+d y^{2}\right\}+\theta(-u)\left\{-d u d \tilde{v}+d y^{2}\right\}
\end{aligned}
$$

This geometry clearly corresponds to taking the two flat halfspaces, $u>0$ and $u<0$ in $R^{3}$ and identifying the points $(u, \tilde{v}, y)=\left(0, \tilde{v}_{0}, y_{0}\right)$ on $u=0^{-}$with the points $(u, \tilde{v}, y)=$ $\left(0, \tilde{v}_{0}+2 \kappa^{2} E f\left(y_{0}\right), y_{0}\right)$ on $u=0^{+}$.

From the solution to the TMG model, (2.7), we can of course recover its two limits - (ghost) Einstein gravity and CSG — or more simply, we can obtain them directly from (2.5). In the case of Einstein gravity, we are more interested in the usual non-ghost theory to compare with previous work; hence, in this limit only, we solve the equations $G_{\mu \nu}=+\kappa^{2} T_{\mu \nu}$ corresponding to $\mu \rightarrow \infty$ with $\kappa^{2} \rightarrow-\kappa^{2}$ :

$$
G_{u u}=R_{u y u y}=-\frac{1}{2} F^{\prime \prime}=\kappa^{2} T_{u u}=\kappa^{2} E \delta(y) \delta(u)
$$

vanishes except on the photon trajectory. Integrating 2.10 we find

$$
F=-2 \kappa^{2} E y \theta(y) \delta(u)+B(u) y+C(u)
$$

corresponding to the $\mu \rightarrow \infty\left(\kappa^{2} \rightarrow-\kappa^{2}\right)$ limit of (2.7). (This solution with $(B, C)=$ $\left(\kappa^{2} E \delta(u), 0\right)$ has appeared previously [5].) The homogeneous $B y+C$ terms can again be removed by (2.8), leaving the interval

$$
d s^{2}=d s_{0}^{2}-2 \kappa^{2} E y \theta(y) \delta(u) d u^{2}
$$

where $F$ now has support on the $\{u=0, y>0\}$ null halfplane. In contrast with TMG $F$ can also be made to have support just on the $y<0$ halfplane by choosing $(B, C)=$ $\left(2 \kappa^{2} E \delta(u), 0\right)$. Both forms of the metric are of course flat outside the source and related by the coordinate transformation, (2.8).

The CSG model is likewise most easily solved directly from (2.5), i.e., from $F^{\prime \prime \prime}=$ $2 \bar{\mu} E \delta(y) \delta(u)$ where $\bar{\mu} \equiv \kappa^{2} \mu$ is the relevant coupling constant. Furthermore, since $C^{\mu \nu}$ is the $D=3$ Weyl tensor, it only determines the metric up to a conformal factor; thus using 
(2.5) or the corresponding limit of the TMG solution involves a particular conformal gauge choice. Here the curvature is

$$
G_{u u}=-\frac{1}{2} F^{\prime \prime}=-\bar{\mu} E(\theta(y) \delta(u)+D(u)) .
$$

There is no preferred choice of $D(u)$, since the curvature cannot in any case be made to vanish at both $y=\infty$ and $y=-\infty$. The corresponding metric (after removing the homogeneous solutions $B y+C$ and up to an arbitrary conformal factor) is

$$
d s^{2}=d s_{0}^{2}+\bar{\mu} E y^{2}(\theta(y) \delta(u)+D(u)) d u^{2} .
$$

\section{Boosting.}

In this section, we obtain the one-photon solutions by an infinite boost of the corresponding static "Schwarzschild" metrics in the same spirit as for $D=4$ gravity [6]. We begin with the usual non-ghost Einstein gravity, whose conical metric for a static mass $m$ [4] we write in the form:

$$
d s^{2}=d s_{0}^{2}+\left(\alpha^{2}-1\right) \frac{(y d x-x d y)^{2}}{\left(x^{2}+y^{2}\right)}
$$

where $\alpha=1-\frac{\kappa^{2} m}{2 \pi}$, and $d s_{0}^{2}=-d t^{2}+d x^{2}+d y^{2}$. We now perform a boost in the $x$ direction with boost parameter $\beta=\tanh \xi$. The first term, $d s_{0}^{2}$, is of course invariant, while in the second term $x$ is replaced by $\tilde{x} \equiv \cosh \xi x-\sinh \xi t$. For large $\xi$ and small $m$ with the energy $E=m \cosh \xi$ fixed, the metric becomes

$$
d s^{2}=d s_{0}^{2}-\kappa^{2} E \frac{e^{\xi}}{2 \pi} \frac{(-y d u+u d y)^{2}}{\left(\frac{e^{\xi}}{2} u\right)^{2}+y^{2}} .
$$

Using the fact that

$$
\lim _{\xi \rightarrow \infty} \frac{e^{\xi}}{2 \pi} \frac{1}{\left(\frac{e^{\xi}}{2} u\right)^{2}+y^{2}}=\frac{1}{\pi} \delta(u) \int_{-\infty}^{\infty} \frac{d s}{s^{2}+y^{2}}=\frac{1}{|y|} \delta(u),
$$

we see that (3.2) reduces to (2.11), the one-photon solution, with $(B, C)=\left(\kappa^{2} E \delta(u), 0\right)$. It is not surprising that we obtained the form of the metric $(F \propto|y| \delta(u))$ symmetric in $y$, since we began with a symmetric metric (3.1) and boosted in $x$.

We now obtain the one-photon TMG metric by boosting the corresponding stationary "Schwarzschild" TMG solution. Actually only the linearized metric for a static source is 
known [7]. This will be sufficient, however, since we take $m \rightarrow 0$ in the infinite boost method. Indeed, the linearized solution is always a sufficient starting point (for Einstein gravity recall the existence of Eddington coordinates in terms of which the Schwarzschild solution is already linear in $m$ ). It is more convenient here to first apply the coordinate transformation $\rho=\frac{r^{1+\frac{\kappa^{2} m}{2 \pi}}}{1+\frac{\kappa^{2} m}{2 \pi}} \approx r+\frac{\kappa^{2} m}{2 \pi} r(\ln r-1)$ to the linearized solution as given in [7]. We then have

$$
d s^{2}=d s_{0}^{2}+\frac{1}{2 \pi} \kappa^{2} m\left\{K_{0}(\mu \rho)\left(d t^{2}+d \rho^{2}+\rho^{2} d \phi^{2}\right)+2 \rho^{2} d \phi^{2}+\frac{2}{\mu}\left(\rho \frac{\partial K_{0}(\mu \rho)}{\partial \rho}+1\right) d t d \phi\right\}
$$

where $K_{0}$ is the modified Bessel function and $d s_{0}^{2}=-d t^{2}+d \rho^{2}+\rho^{2} d \phi^{2}$. [In the limit $\mu \rightarrow \infty$, we of course regain the (linearized) "ghost" version of (3.1) in polar coordinates.] For a boost with large $\xi$

$$
\begin{gathered}
t, x \rightarrow \frac{e^{\xi}}{2} u, \quad \rho^{2} \rightarrow \tilde{\rho}^{2} \equiv\left(\frac{e^{\xi}}{2}\right)^{2} u^{2}+y^{2} \\
d t^{2} \rightarrow\left(\frac{e^{\xi}}{2}\right)^{2} d u^{2}, \quad \rho^{2} d \phi^{2} \rightarrow\left(\frac{e^{\xi}}{2}\right)^{2} \frac{(u d y-y d u)^{2}}{\tilde{\rho}^{2}}, \quad d t d \phi \rightarrow\left(\frac{e^{\xi}}{2}\right)^{2} \frac{(u d y-y d u) d u}{\tilde{\rho}^{2}} .
\end{gathered}
$$

For large $\xi$ and small $m$ with $E=m \cosh \xi$ fixed, eqn. (3.4) then becomes

$$
d s^{2}=d s_{0}^{2}+\frac{1}{\pi} \kappa^{2} E\left(\frac{e^{\xi}}{2}\right)\left\{K_{0}(\mu \tilde{\rho}) d u^{2}+\frac{(u d y-y d u)^{2}}{\tilde{\rho}^{2}}+\frac{1}{\mu}\left(\tilde{\rho} \frac{\partial K_{0}(\mu \tilde{\rho})}{\partial \tilde{\rho}}+1\right) \frac{(u d y-y d u) d u}{\tilde{\rho}^{2}}\right\} .
$$

To evaluate this limit, we recall the identity generalizing (3.3),

$$
\lim _{\xi \rightarrow \infty} \frac{e^{\xi}}{2} W\left(\tilde{\rho}^{2}, y\right)=\delta(u) \int_{-\infty}^{\infty} W\left(s^{2}+y^{2}, y\right) d s, \quad \tilde{\rho}^{2} \equiv\left(\frac{e^{\xi}}{2}\right)^{2} u^{2}+y^{2}
$$

provided $\tilde{\rho} W\left(\tilde{\rho}^{2}, y\right) \rightarrow 0$ as $\tilde{\rho} \rightarrow \infty$. It follows from the $\delta$-function in (3.7) that only the coefficient of $d u^{2}$ in (3.6) survives the limit. Upon performing the required integration in (3.7), we check that the infinite boost limit of (3.6) is just the one-photon TMG solution, (2.7), with $B=-\kappa^{2} E \delta(u), C=\frac{\kappa^{2} E}{\mu} \delta(u)$; again $B$ and $C$ can be transformed away by $(2.8)$.

Finally, we remark that there is no direct boost approach to CSG since $C_{\mu}^{\mu}$ vanishes identically, and hence $C^{\mu \nu}$ can only couple to a traceless stress tensor, such as a lightlike particle; TMG can thus be thought of as a "regularization" permitting us also to obtain the CSG metric by first boosting and then limiting to CSG. 


\section{Multiphoton Solutions.}

We now derive multiphoton solutions to TMG, as well as to Einstein gravity. Surprisingly, we will find that there exist geometries, even in the dynamical context of TMG, corresponding to some superpositions of freely moving, noncollinear photons. We therefore begin by describing the kinematics of $n$ photons with energies $E_{i}, i=1, \ldots, n$ moving along lightlike flat space geodesics, i.e., straight lines in Cartesian coordinates with null tangent vectors. Every photon's worldline can be obtained by a rotation and translation, $x^{\mu} \rightarrow x_{i}^{\mu}=\left(\Omega_{i} x\right)^{\mu}+a_{i}^{\mu}$, of some base trajectory $u \equiv t-x=0, y=0$. The corresponding energy-momentum tensor is $T_{i \mu \nu}=E_{i} \delta\left(u_{i}\right) \delta\left(y_{i}\right) \partial_{\mu} u_{i} \partial_{\nu} u_{i}$. The solution to the associated, one-photon, equation of motion $E_{\mu \nu}=-\kappa^{2} T_{i \mu \nu}$ is correspondingly obtained by applying the rotation and translation to the base source one-photon solution of Section 2, yielding $g_{i \mu \nu}=\eta_{\mu \nu}+2 \kappa^{2} E_{i} f\left(y_{i}\right) \delta\left(u_{i}\right) \partial_{\mu} u_{i} \partial_{\nu} u_{i}$. To understand the $n$-photon system, first consider two photons whose energy-momentum tensor is given by the sum of the individual onephoton contributions, $T_{\mu \nu}=T_{1 \mu \nu}+T_{2 \mu \nu}$. For parallel photons, which we can assume to be moving along the $x$-axis, each of their one-photon solutions, $g_{1 \mu \nu}$ and $g_{2 \mu \nu}$, are of the form (2.1). In this case $E_{\mu \nu}$ linearizes* $\left(E_{\mu \nu}\left[h_{1 \alpha \beta}+h_{2 \alpha \beta}\right]=E_{\mu \nu}\left[h_{1 \alpha \beta}\right]+E_{\mu \nu}\left[h_{2 \alpha \beta}\right]\right)$, and the two-photon solution is simply the superposition of the one-photon solutions, $h_{\mu \nu}=h_{1 \mu \nu}+h_{2 \mu \nu}$. Superposition of parallel photons also holds in $D=4$ gravity. However, in $D=3$ the one-photon solutions will be seen to superpose for some nonparallel photon configurations as well. The local functional $E_{\mu \nu}$ obeys $E_{\mu \nu}\left[h_{\alpha \beta}=0\right]=0$. Therefore, if the two regions on which $h_{1 \alpha \beta}$ and $h_{2 \alpha \beta}$ have support are disjoint, then $E_{\mu \nu}\left[h_{1 \alpha \beta}+h_{2 \alpha \beta}\right]=E_{\mu \nu}\left[h_{1 \alpha \beta}\right]+E_{\mu \nu}\left[h_{2 \alpha \beta}\right]$ implying again that the two-photon solution is given by $h_{\mu \nu}=h_{1 \mu \nu}+h_{2 \mu \nu}$. [In $D=4$, the one-photon metric [6] has support on an entire null hyperplane; since two non-parallel hyperplanes necessarily intersect, only parallel photons can be superposed there.] Now for general $n$, superposing the one-photon solutions, $g_{\mu \nu}=\eta_{\mu \nu}+\sum_{i=1}^{n} h_{i \mu \nu}$, will yield the $n$-photon solution provided every pair of photons is either parallel or oriented such that the respective halfplanes $\left(u_{i}=0, y_{i}>0\right)$ on which the $h_{i \mu \nu}$ have support are disjoint.

For two non-colliding photons in Einstein gravity, this method of superposition can be used to construct the solution for every orientation of the particles. For two photons in TMG as well as for $n>2$ photons in any of the models, not all orientations of the photons

* We work with $h_{i \mu \nu} \equiv g_{i \mu \nu}-\eta_{\mu \nu}$ rather than $g_{i \mu \nu}$ in order to avoid "overcounting" their $\eta_{\mu \nu}$ parts. 
satisfy the criteria for superposition; in fact, no orientation of $n>4$ mutually nonparallel photons satisfies them in TMG or in Einstein gravity (as is geometrically clear). Consider first two photons in Einstein gravity. We can go to their center-of-momentum frame where we take the two particles to be moving antiparallel in the $x$-direction with equal energies $E$ along the trajectories $u=y=0$ and $v=0, y=a \neq 0$. In the notation of the previous paragraph, $u_{1}=u, v_{1}=v, y_{1}=y$ and $u_{2}=v, v_{2}=u, y_{2}=a-y$. As discussed in Section 2 , we can construct the Einstein gravity solution for one photon, $h_{\mu \nu}$, moving along the $u=y=0$ trajectory to have support on either the $\{u=0, y>0\}$ or $\{u=0, y<0\}$ null halfplanes by appropriate choice of homogeneous solutions. For two photons, if we make the following choice of homogeneous solutions, $\left(B_{1}, C_{1}\right)=\left(2 \kappa^{2} E \theta(a) \delta(u), 0\right)$ and $\left(B_{2}, C_{2}\right)=\left(2 \kappa^{2} E \theta(a) \delta(v), 0\right), h_{1 \mu \nu}$ and $h_{2 \mu \nu}$ will have support on the disjoint halfplanes, $\{u=0,(\operatorname{sgn} a) y<0\}$ and $\{v=0,(\operatorname{sgn} a)(y-a)>0\}$, allowing us to superpose the one-photon solutions to give the two-photon solution:

$$
d s^{2}=d s_{0}^{2}-2 \kappa^{2} E\left\{y(\theta(y)-\theta(a)) \delta(u) d u^{2}+(a-y)(\theta(a-y)-\theta(a)) \delta(v) d v^{2}\right\} .
$$

We now give an example in which the maximal number (four) of mutually non-parallel photons in TMG superpose, and construct their exact solution. Let two of the particles be antiparallel-moving in the $x$-direction with energies $E_{1}$ and $E_{2}$, and the other two antiparallel in the $y$-direction with energies $E_{3}$ and $E_{4}$. Their trajectories and null halfplanes are given respectively by $(t=x, y=0),\{t=x, y>0\} ;(t=-x, y=a),\{t=-x, y<a\}$;

$(t=y, x=b),\{t=y, x<b\} ;(t=-y, x=-c),\{t=-y, x>-c\}$. It is not difficult to check that if $a, b, c$ are all negative, the four halfplanes are disjoint, and the four-photon solution can be obtained by superposing these four one-photon solutions:

$$
\begin{aligned}
d s^{2}= & d s_{0}^{2}+2 \kappa^{2}\left\{E_{1} f(y) \delta(t-x)(d t-d x)^{2}+E_{2} f(-y+a) \delta(t+x)(d t+d x)^{2}\right. \\
& \left.+E_{3} f(-x+b) \delta(t-y)(d t-d y)^{2}+E_{4} f(x+c) \delta(t+y)(d t+d y)^{2}\right\}
\end{aligned}
$$

\section{Geometrical Approach.}

In this section we obtain the Einstein gravity solutions of the previous sections by gluing together patches of three-dimensional Minkowski space with Poincare transformations. (In TMG, since spacetime is not flat outside sources, this is not possible.) Solutions in Einstein gravity were obtained this way in [4] for timelike sources, and in [8] for tachyonic ones. Solutions for lightlike sources can be obtained from the former by taking the limit 
$\beta \rightarrow 1$ and $m \rightarrow 0$ with the energy fixed. We recall that a static particle of mass $m(\leq 2 \pi)$ is described by the conical spacetime constructed by first extracting a wedge of angle $m$ (where we are setting $\kappa^{2}=1$ ) from the $x-y$ plane with vertex at the origin coinciding with the particle's position. Points along the two edges are then identified by $\mathbf{x}^{\prime}=\Omega_{m} \mathbf{x}$ where $\Omega_{m}$ is a rotation matrix

$$
\Omega_{m}=\left(\begin{array}{ccc}
1 & 0 & 0 \\
0 & \cos m & \sin m \\
0 & -\sin m & \cos m
\end{array}\right)
$$

in the coordinate system $\mathbf{x}=(t, x, y)$. To obtain the solution for a particle moving with speed $\beta=\tanh \xi$ in the positive $x$ direction and located at spacetime point $\mathbf{a}$, we boost and translate the conical spacetime yielding the identification

$$
\mathbf{x}^{\prime}=\mathbf{a}+\left(\Lambda_{\xi} \Omega_{m} \Lambda_{\xi}^{-1}\right)(\mathbf{x}-\mathbf{a})
$$

where $\Lambda_{\xi}$ is the boost,

$$
\Lambda_{\xi}=\left(\begin{array}{ccc}
\cosh \xi & \sinh \xi & 0 \\
\sinh \xi & \cosh \xi & 0 \\
0 & 0 & 1
\end{array}\right) .
$$

For a photon we take the infinite boost limit with the energy $E=m \cosh \xi$ fixed. With $\mathbf{a}=\mathbf{0}$, (5.2) then becomes

$$
\mathbf{x}^{\prime}=N_{E} \mathbf{x}, \quad N_{E}=\left(\begin{array}{ccc}
1+\frac{1}{2} E^{2} & -\frac{1}{2} E^{2} & E \\
\frac{1}{2} E^{2} & 1-\frac{1}{2} E^{2} & E \\
E & -E & 1
\end{array}\right)
$$

which in light cone coordinates corresponds to

$$
\left(u^{\prime}, v^{\prime}, y^{\prime}\right)=\left(u, v+2 E y+E^{2} u, y+E u\right) .
$$

The matrices $N_{E}$ represent all Lorentz transformations which leave the worldline $u=y=0$ invariant; they therefore play the same role for a lightlike particle as the rotations $\Omega_{m}$ play in the case of a static particle. If one now makes a cut along the $u=0, y>0$ halfplane, and repastes with the identification (5.5), one obtains the pure gravity limit of the one-photon solution, (2.9), derived earlier. We note that one can also identify distinct halfplanes containing the worldline which are mapped into one another by $N_{E}$. This leads to the class of solutions known as null orbifolds which have been discussed previously in the context of string theory [9]. 
A system of particles can be described by composing the respective one-particle identifications. For two particles, this yields

$$
\begin{aligned}
\mathbf{x}^{\prime \prime} & =\mathbf{a}_{\mathbf{1}}+\Lambda_{1} \Omega_{1} \Lambda_{1}^{-1}\left(\mathbf{x}^{\prime}-\mathbf{a}_{\mathbf{1}}\right) \\
& =\mathbf{a}_{\mathbf{1}}+\Lambda_{1} \Omega_{1} \Lambda_{1}^{-1}\left(\left(\mathbf{a}_{\mathbf{2}}-\mathbf{a}_{\mathbf{1}}\right)+\Lambda_{2} \Omega_{2} \Lambda_{2}^{-1}\left(\mathbf{x}-\mathbf{\mathbf { a } _ { 2 }}\right)\right) .
\end{aligned}
$$

This in turn is equivalent to the identification, (5.2), for a single composite particle where $T \equiv \Lambda \Omega_{m} \Lambda^{-1}=\Lambda_{1} \Omega_{1} \Lambda_{1}^{-1} \Lambda_{2} \Omega_{2} \Lambda_{2}^{-1} . m$ is the total mass of the system, and $\Lambda$ is some boost. Taking the trace then yields

$$
\begin{aligned}
\cos m & =\frac{1}{2}\left(\operatorname{Tr} \Omega_{m}-1\right)=\frac{1}{2}(\operatorname{Tr} T-1) \\
& =\frac{1}{2}\left(\operatorname{Tr}\left(\Lambda_{1} \Omega_{1} \Lambda_{1}^{-1} \Lambda_{2} \Omega_{2} \Lambda_{2}^{-1}\right)-1\right) .
\end{aligned}
$$

For two photons both moving in the positive $x$-direction with energies $E_{1}$ and $E_{2}$, this implies $T=N_{E_{1}} N_{E_{2}}=N_{E_{1}+E_{2}}$. From (5.7), we find as expected that $m=0$. For photons moving antiparallel in their center-of-momentum frame each with energy $E$, the matrix becomes $T=\left(\Omega_{\pi} N_{E} \Omega_{-\pi}\right) N_{E} ;(5.7)$ then implies

$$
\cos m=1-2 E^{2}+\frac{1}{2} E^{4} .
$$

For small $E$, this reduces to $m=2 E$, the usual flat space mass addition formula. In order for the mass $m$ to be real (non-tachyonic), the right hand side of (5.8) must be in the interval $[-1,1]$. This in turn places an upper bound on the energy: $E<E_{\max }=2$.

As a last application, consider a mixed system consisting of a photon and a particle of finite mass $M$. Even though we did not obtain the analytic form for the metric describing this system, we can still describe the solution geometrically. In a frame in which the massive particle is at rest and the photon is moving in the positive $x$ direction with energy $E$, we have $T=N_{E} \Omega_{M}$. Taking the trace, we find that the mass $m$ of the composite system is given by

$$
\cos m=\cos M-(\sin M) E+\frac{1}{4}(1-\cos M) E^{2} .
$$

For small $M$ and $E$, this again coincides with the flat space formula, $m^{2}=M^{2}+2 M E$. As before, the condition that the total mass be real places an upper bound on $E$

$$
E<E_{\max }=2 \frac{\sin M+\sqrt{2(1-\cos M)}}{1-\cos M} .
$$

As expected we see that as $M \rightarrow 0, E_{\max } \rightarrow \infty$. On the other hand, as $M$ approaches its maximal value of $2 \pi$, observe that $E_{\max } \rightarrow 0$ (since $\sin M<0$ ), i.e., no photon is permitted in this limiting cylindrical geometry. 


\section{Discussion.}

We have obtained a series of explicit solutions for both the general nonlinear TMG model and its limiting cases of Einstein gravity and pure Chern-Simons gravity in $D=3$, all generated by (at least) one null matter source. These solutions have a number of possible applications. First, as we will discuss elsewhere [3], the single photon metric can be used to obtain the eikonal gravitational scattering amplitude for a timelike, massive particle colliding at small angles with the rapid one, following the methods of [1]; because the plane waves are always impulsive, the metric can written as a (singular) pure gauge, resulting in an Aharonov-Bohm type of scattering. Secondly, since our solutions involve moving particles and hence have nonzero orbital angular momentum, they will permit us to analyze [10] the conditions under which closed timelike curves can be present both in

Einstein gravity (as well as TMG) for quite different sources than those used in previous work [4], [8], [11].

\section{Acknowledgements}

We thank J. McCarthy for useful discussions in the early stages of this work. S. D. thanks T. Dereli for informative correspondence on the CSG problem.

This work was supported in part by NSF Grant No. PHY-88-0451. 


\section{References}

[1] T. Dray and G. 't Hooft, Nucl. Phys. B253 (1985) 173; G. 't Hooft, Phys. Lett. B198 (1987) 61; Nucl. Phys. B304 (1988) 867.

[2] S. Deser, R. Jackiw, S. Templeton, Ann. Phys. 140 (1982) 372.

[3] S. Deser, J. McCarthy, A. Steif, "Planck Scale Scattering in $2+1$ Gravity Theories", in preparation.

[4] S. Deser, R. Jackiw, G. 't Hooft, Ann. Phys. 152 (1984) 220.

[5] A. Cappelli, M. Ciafaloni, and P. Valtancoli, Phys. Lett. B273 (1991) 431.

[6] P. C. Aichelburg and R. U. Sexl, J. Gen. Rel. Grav. 2 (1971) 303.

[7] S. Deser, Phys. Rev. Lett. 64 (1990) 611.

[8] S. Deser, R. Jackiw, and G. 't Hooft, Phys. Rev. Lett. 68 (1992) 267.

[9] G. Horowitz and A. Steif, Phys. Lett. B258 (1991) 91.

[10] S. Deser and A. Steif, "Time Machines from Lightlike Sources?," in preparation.

[11] J. Gott, Phys. Rev. Lett. 66 (1991) 1126; A. Ori, Phys. Rev. D 44 (1991) R2214; C. Cutler, Phys. Rev. D 45 (1992) 487; S. Carroll, E. Farhi, and A. Guth, Phys. Rev. Lett. 68 (1992) 263, (E)3368; MIT preprint CTP-2117 (1992); G. 't Hooft, Class. Quantum Grav. 9 (1992) 1335; D. Kabat, MIT preprint CTP-2034 (1992). 